\title{
Characterization of Localized Degradation in Reverse-Biased GaN HEMTs by Scanning Transmission Electron Microscopy and Electron Holography
}

\author{
David A. Cullen*, David J. Smith**, Antonio Stocco***, Gaudenzio Meneghesso***, Enrico \\ Zanoni*** and Martha R. McCartney** \\ * School of Materials, Arizona State University, Tempe, AZ 85287-8706 \\ ** Department of Physics, Arizona State University, Tempe, AZ 85287-1504 \\ *** Department of Information Engineering, University of Padova, Padova, Italy 35131
}

The $\mathrm{AlGaN} / \mathrm{GaN}$ heterostructure provides the basis for the current generation of high electron mobility transistor (HEMT) devices. Due to their large band gap, high thermal conductivity and high saturation drift velocity; GaN-based devices have great potential as high-power, high-frequency devices and switches [1]. The current challenge facing these high-performance devices has been maintaining device reliability and stability. Current research is focused on identifying possible failure modes caused by defect formation arising from the inverse piezoelectric effect [2]. High electric fields combined with the piezoelectric nature of the barrier material result in very high localized stress at the gate edge, which can lead to the formation of defects and eventual device degradation [3]. This ongoing study is utilizing the phase sensitivity of electron holography to compare the two-dimensional electrostatic potential at the gate edge in stressed and unstressed devices to investigate electrical degradation caused by reverse-bias stress testing

Reverse-bias step tests were performed on HEMT devices with different gate lengths. Electroluminescence (EL) microscopy was used to detect light emission from high energy electrons injected into the semiconductor from the gate [4]. The appearance of these localized "hot spots" at the gate edge was correlated with an increase in gate leakage current. EL images, such as the one shown in Fig. 1(a), were used to map out the locations of defective regions where electron injection had occurred. Samples suitable for TEM and holography observations were prepared at the identified sites of these hot spots using focused-ion-beam (FIB) lift-out. Transistor cross-sections were lifted out using a forked probe tip, and then mounted onto a copper washer for backside milling using a shortcut press as shown in Fig. 2(a). Membranes were then thinned to electron transparency from the substrate side with a final Ga ion-beam energy of $5 \mathrm{keV}$. Bright field TEM (Fig. 2(b)) and high-angle annular-dark-field STEM (Fig. 3(a)) images of the gate edge show a V-shaped defect located in the same region where a hot spot was previously detected. As shown in Fig. 3(b), EDX linescans in the region of the cavity between the gate and V-defect revealed a mixture of Al, Si, Ga, and Ti. This defective zone could provide a current leakage path that would eventually cause device failure [5].

[1] H. Okamura, Japan. J. Appl. Phys. 45 (2006) 7565.

[2] J. A. del Alamo and J. Joh, Microelectron. Reliab. 49 (2009) 1200.

[3] S. Y. Park et al., Microelectron. Reliab. 49 (2009) 478.

[4] E. Zanoni et al., IEEE Electron Device Lett. 30 (2009) 427.

[5] This work was supported by a contract from Wright-Patterson Air Force Base (Monitor: C. Bozada). The authors gratefully acknowledge the use of facilities within the John M. Cowley Center for High Resolution Electron Microscopy at Arizona State University. 


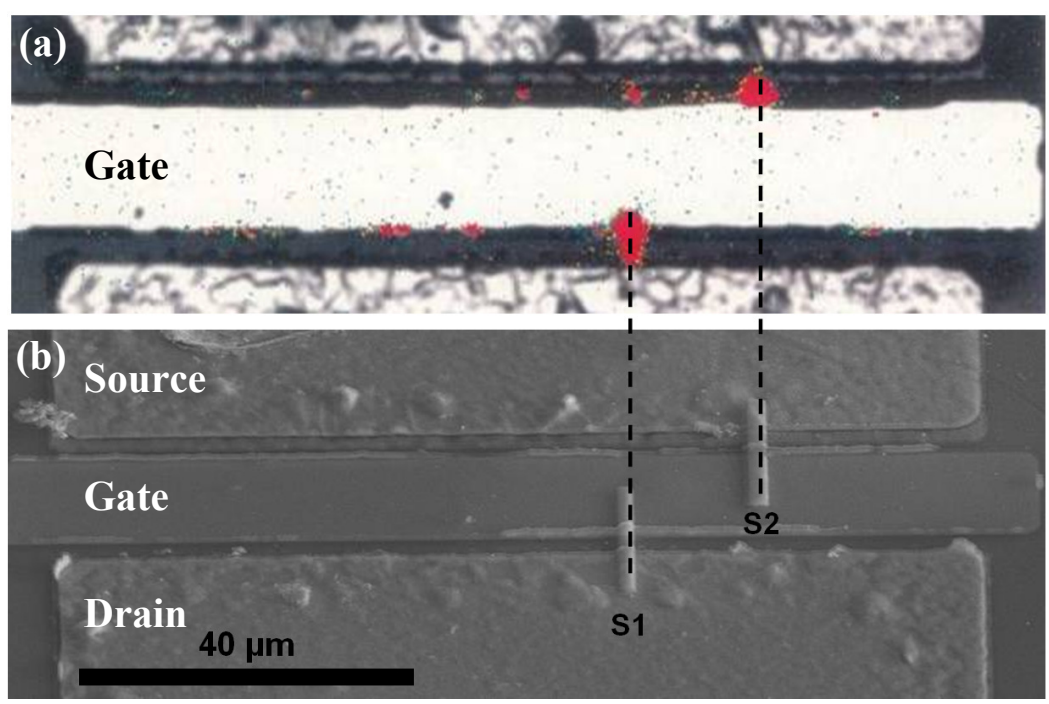

Figure 1. (a) EL image recorded during high-stress reverse biasing. Light emission occurred in localized regions at the gate edge where defects are believed to provide a leakage path for hot electrons. (b) SEM image of identical area; Pt bars were deposited over the localized hot spots in preparation for FIB cutting and subsequent lift-out.
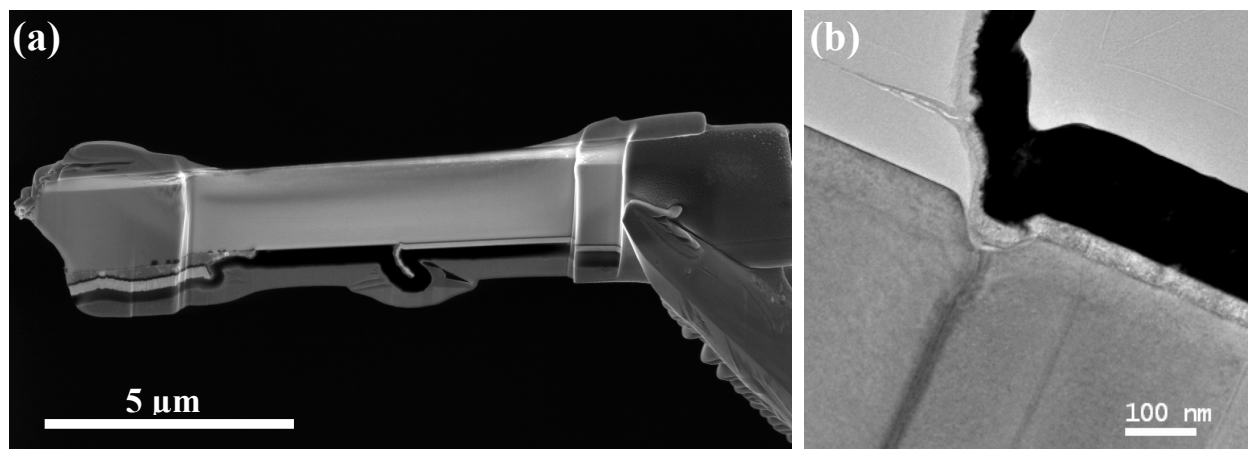

Figure 2. (a) SEM image of FIB lift-out specimen prepared from the region labeled S1 in Figure 1. The cross-section was mounted for backside milling using the Omniprobe short-cut press to avoid curtaining effects during FIB thinning. (b) Bright-field TEM image of the gate edge.
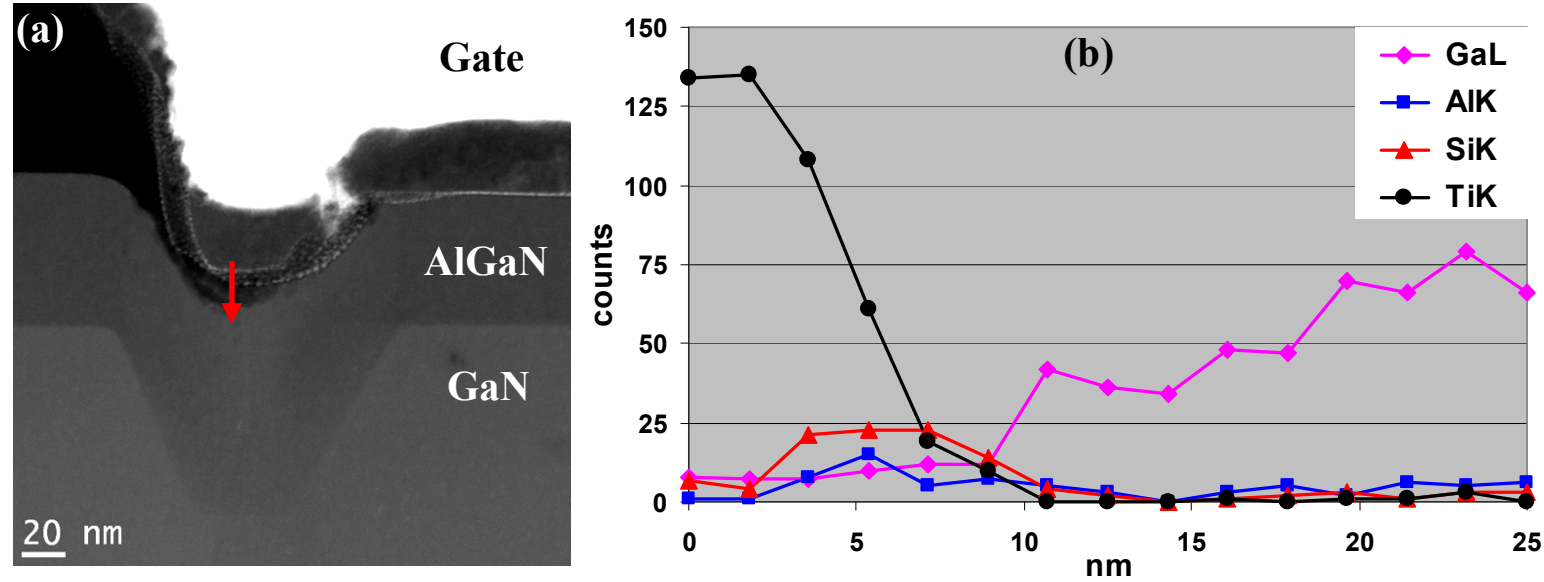

Figure 3. (a) HAADF-STEM image of gate edge, showing the presence of a V-shaped defect that was responsible for the gate leakage current visualized in the EL image in Figure 1. (b) EDX linescan along the arrow in the STEM image, indicating the cavity below the gate contains a mixture of $\mathrm{Si}, \mathrm{Al}, \mathrm{Ti}$, and $\mathrm{Ga}$. 